\section{Proaktive Kontrolle statt reaktive Therapie}

$\mathrm{N}$ eben der Akuttherapie von Ekzemschüben wird - analog zur Asthmatherapie - auch bei der atopischen Dermatitis ein erfolgreiches Langzeitmanagement angestrebt. Im Frühjahr 2009 wurde dazu ein neues Behandlungskonzept zugelassen: die proaktive Erhaltungstherapie mit dem topischen Calicineurininhibitor Tacrolimus (Protopic ${ }^{\circledR}$ ). Dabei wird Tacrolimus-Salbe - ohne Vorliegen akuter Ekzeme - längerfristig zweimal pro Woche auf zuvor befallene Hautareale aufgetragen. Rationale dieses Vorgehens sind neue Erkenntnisse zum Pathomechanismus der atopischen Dermatitis, die deutlich machen, dass auch nach dem Abklingen eines akuten Ekzemschubs eine subklinische Entzündung der Haut bestehen bleibt, die die Grundlage für zukünftige Exazerbationen darstellt.

Dass mit dem neuen Therapiekonzept eine effektive Kontrolle der Erkrankung erreicht werden kann, bestätigen die Ergebnisse der CONTROL-Kinderstudie (Thaçi D et al. Brit J Dermatol
2008; 159: 1348-56): 267 Kinder im Alter zwischen zwei und 15 Jahren mit atopischer Dermatitis wendeten Tacrolimus-Salbe im Anschluss an eine intensive Initialtherapie zweimal wöchentlich an. Sie profitierten von der proaktiven Erhaltungstherapie mit einer si-

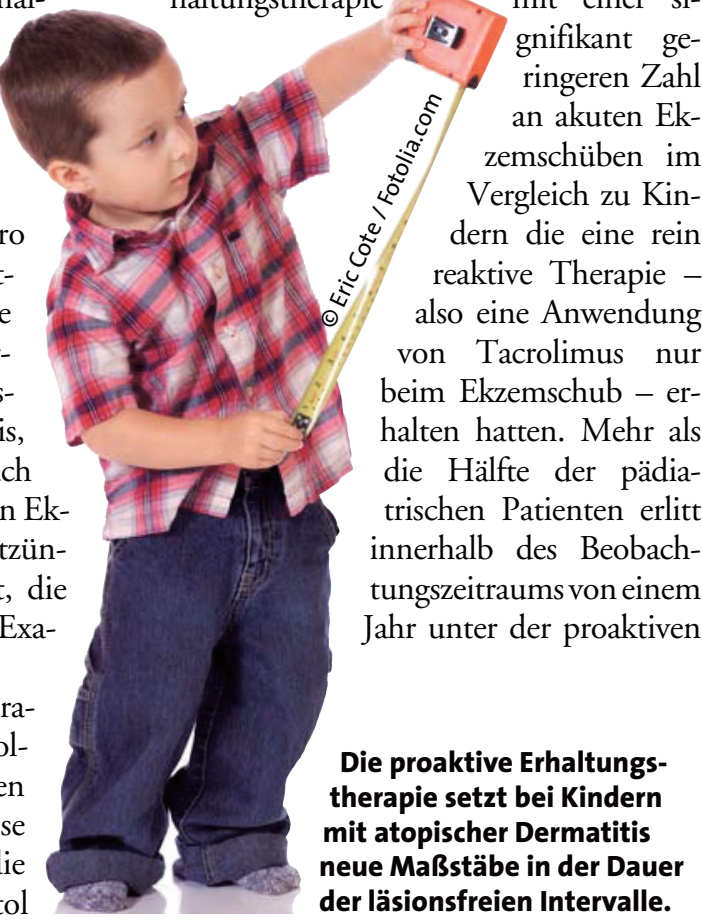

Therapie keinen einzigen Ekzemschub, und die Zeit bis zum Auftreten des ersten Schubes war unter proaktiver Therapie signifikant länger als unter reaktiver Therapie (36 versus 217 Tage).

Die proaktive Therapie verursachte dabei nicht mehr Kosten als die herkömmliche Behandlung, wie eine aktuelle erneute Auswertung der Daten der CONTROL-Studie ergab (Reitamo S et al. J Dermatol Treat 2010; 21: 3444): Der Salbenverbrauch war in beiden Studienarmen vergleichbar (1,0 g/d vs. $1,1 \mathrm{~g} / \mathrm{d})$. Eine analoge Studie mit 257 Erwachsenen kam zu vergleichbar positiven Ergebnissen.

Tacrolimus-Salbe wird seit zehn Jahren zur Therapie der atopischen Dermatitis eingesetzt. Entsprechend umfangreiche Daten bestätigen die gute Verträglichkeit und das ausgezeichnete Sicherheitsprofil auch in der Langzeittherapie. Dementsprechend betont eine gerade publizierte Übersichtsarbeit (Thaçi D et al. Clin Dermatol 2010; 28: 52-6) nochmals, dass es bisher keinerlei Hinweise auf ein erhöhter Risiko für maligne Erkrankungen unter der Therapie mit topischen Calcineurininhibitoren gibt.

Nach Informationen von Astellas Pharma, München

Dabei zeigte sich, dass Rupatadin nicht nur die Symptome gelindert hatte, sondern auch in anderen Kategorien der Lebensqualität, wie z. B. Schlafstörungen, psychologische Belastung oder Intensität der täglichen Aktivitäten, zu Verbesserungen geführt hatte.

Das gute kardiale Sicherheitsprofil von Rupatadin schließlich konnte in einer gerade publizierten randomisierten, doppelblinden und plazebokontrollierten Studie aus Spanien erneut bestätigt werden (Donado E et al. Br J Clin Pharmacol 2010; 69: 401-10): Rupatadin führte weder in der zugelassenen $10-\mathrm{mg}$ Dosierung noch in einer zehnfach erhöhten Dosis zu einer QT/QTc-Streckenverlängerung.

Nach Informationen von Merckle Recordati, Ulm 